\title{
6. Einführung in das Psych-Entgeltgesetz (PEPP)
}

\author{
Eric Hahn und Albert Diefenbacher
}

Die Vergütung der Leistungen psychiatrischer Kliniken und Abteilungen erlebt derzeit mit der Einführung des Psych-Entgeltgesetzes einen grundlegenden Wandel, der auch für Sie als in der Psychiatrie oder Psychosomatik tätige Ärzte eine nicht zu unterschätzende Bedeutung haben wird. Ein grundsätzliches Verständnis der Logik des neuen Pauschalierenden Entgeltsystems für Psychiatrie und Psychosomatik (PEPP) ist dabei von Beginn Ihrer Ausbildung und klinischen Arbeit an unabdingbar, da eine auf dieser Logik aufbauende konsistente, vollständige Dokumentation der relevanten Prozeduren und Diagnosen die Vorrausetzung für eine leistungsgerechte Vergütung darstellen wird. Auch wenn alle Berufsgruppen kodieren sollen, wird die entgeltrelevante Auflistung von Haupt- und Nebendiagnosen und der kodierten Prozeduren in der Verantwortung der behandelnden Ärzte liegen.

Das Kapitel erleichtert Ihnen den Einstieg in das mit Einführung des neuen Entgeltsystems notwendige ökonomische, aber klinisch relevante Denken und macht Sie mit den wichtigsten Begriffen und Kodierungsregeln vertraut. Da PEPP als ein „lernendes System“ angelegt ist und Sie sich im Sinne eines „lebenslangen Lernens" an die jährlichen Veränderungen anpassen werden, lohnt sich Ihre frühe Investition in das Verständnis von PEPP besonders.

\section{Hintergrund und gesetzlicher Rahmen}

Seit 1991 rechnen Krankenhäuser die Behandlungen ihrer Patienten in der Psychiatrie nach Tagessätzen auf Grundlage der Psychiatrie-Personalverordnung (Psych-PV) ab. War der anhand der Psych-PV gesetzlich geregelte und von der Belegung abhängige Personalbedarf einer Klinik oder Station nicht erfüllt, konnte er bei den Verhandlungen mit den Krankenkassen geltend gemacht werden, um höhere Budgets zu erzielen. Die verhandelten Tagessätze für stationäre oder teilstationäre Behandlungen waren dabei von der Liegedauer, aber auch von der psychiatri- 
schen Diagnose weitgehend unabhängig. Der Erlös wurde aus der Multiplikation der Pflegetage mit dem Tagespflegesatz berechnet und konnte für Kliniken verschiedener Bundesländer sehr unterschiedlich sein.

2009 wurde entschieden, die Psych-PV durch ein leistungsorientiertes Vergütungssystem abzulösen, wobei als Abrechnungsgrundlage das in den somatischen Fächern bekannte DRG-System (Diagnosis Related Groups) als Vorbild dienen sollte. Allerdings zeigten sich die in den somatischen Fächern verwendeten Fallpauschalen als nicht geeignet, die Behandlung von psychisch Kranken abzubilden. Daher wurde entschieden, dass PEPP sich auf Tagespauschalen beziehen solle. Die Berechnungsgrundlage für die neuen Entgelte bilden dabei seit 2010 die Versorgungsdaten aus den Kliniken für Psychiatrie, Kinder- und Jugendpsychiatrie und Psychosomatik. Eine Gruppe von sogenannten „Kalkulationshäusern" kodieren ihre erbrachten Leistungen einschließlich der Gesamtkosten der Behandlung pro Patient und Tag, anhand eines speziellen Operationen- und Prozedurenschlüssels (OPS), die vom Deutschen Institut für medizinische Dokumentation und Information (DIMDI) herausgegeben werden.

Anhand der Falldaten, den Liegezeiten und den zugehörigen OPS, die an das Institut für das Entgeltsystem im Krankenhaus (InEK) übermittelt werden, erfolgt die Kalkulation und Veröffentlichung von liegezeitabhängigen Vergütungsstufen nach gruppierungsrelevanten Diagnosegruppen.

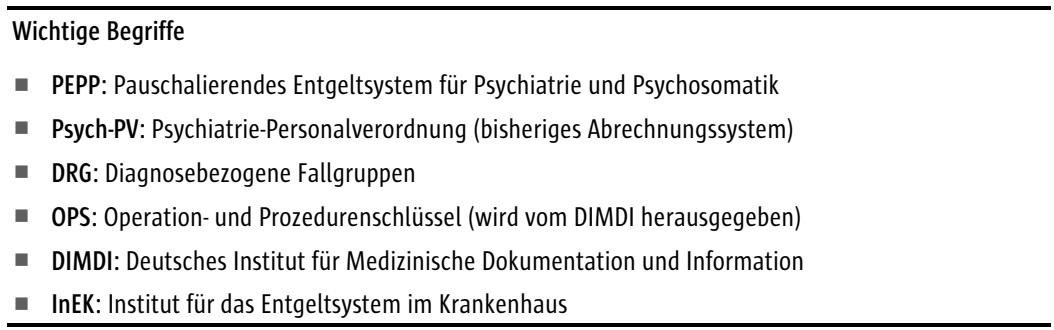

\section{Was sind "PEPPs" denn nun genau?}

PEPP steht wie schon besprochen für Pauschalierendes Entgeltsystem für Psychiatrie und Psychosomatik. Etwas verwirrend wird gleichzeitig auch von „PEPPs“ gesprochen, womit diagnoseorientierte Fallgruppen gemeint sind, die unterschiedliche Merkmale wie z.B. Diagnosegruppen, den Leistungsbereich, das Alter, die Betreuungsintensität und die Gesamtliegedauer zusammenfassen. Diese Zuordnungen zu den PEPPs entscheiden, welchen Erlösbetrag eine psychiatrische Abteilung für einen Behandlungsfall am Ende eines stationären Aufenthaltes bei den Krankenkassen bzw. Kostenträgern geltend machen wird. Aktuell sind im PEPP-Katalog 7 Prä-PEPPs; 14 PEPPs für die vollstationäre psychiatrische Behandlung, 7 PEPPs für die Kinder- und Jugendpsychiatrie, 6 PEPPs für die psychosomatische Behandlung und 7 PEPPs für die tagesklinische Therapie aufgeführt. Um die Übersichtlichkeit zu bewahren, wird sich dieses Kapitel primär auf die Behandlung von Erwachsenen und die vollstationäre psychiatrische Behandlung beziehen. 
Tabelle 4 gibt Ihnen zunächst eine Übersicht über die Diagnosegruppen als Grundlage der Basis-PEPPs für die Psychiatrie. Wie Sie erkennen werden, orientieren sich die meisten, aber nicht alle Diagnosegruppen an den psychiatrischen ICD-10 F-Kategorien. Die für die stationäre Behandlung in der Erwachsenenpsychiatrie relevantesten Gruppen sind fett markiert.

Tab. 4 Gruppierungsrelevante Diagnosegruppen (Basis-PEPP)

\begin{tabular}{|l|l|}
\hline 1 & Intelligenzstörungen, tief greifende Entwicklungsstörungen \\
\hline 2 & Psychische und Verhaltensstörungen durch psychotrope Substanzen \\
\hline 3 & Schizophrenie, schizotype und wahnhafte Störungen \\
\hline 4 & Affektive Störungen \\
\hline 5 & Phobische, andere Angst- oder Zwangsstörungen \\
\hline 6 & Somatoforme oder andere neurotische Störungen \\
\hline 7 & Dissoziative Störungen \\
\hline 8 & Sexuelle Funktionsstörungen oder Störungen der Geschlechtsidentität \\
\hline 9 & Nichtorganische oder organische Schlafstörungen \\
\hline 10 & Ess- oder Fütterstörungen \\
\hline 11 & Hyperkinetische Störungen, Störungen des Sozialverhaltens \\
\hline 12 & Reaktionen auf schwere Belastungen oder Störungen im Wochenbett \\
\hline 13 & Abnorme Gewohnheiten und Störungen der Impulskontrolle \\
\hline 15 & Persönlichkeitsstörungen oder andauernde Persönlichkeitsänderungen \\
\hline 15 & Organische Störungen, degenerative Krankheiten des Nervensystems \\
\hline 13 & zerebrovaskuläre Krankheiten oder Thiaminmangel \\
\hline 13 & andere psychosomatische Störungen \\
\hline 15 andere neuropsychiatrische Symptome \\
\hline 15
\end{tabular}

\section{Wie werden PEPPs kodiert?}

Die Kodierung von PEPPs erfolgt nach einen einfachen Muster und ist immer fünfstellig.

Die ersten beiden Stellen der PEPP-Bezeichnung beschreiben dabei die Strukturkategorie. Die 1. Stelle kann entweder mit $\mathrm{P}=$ vollstationär oder $\mathrm{T}=$ teilstationär kodiert werden. Die 2. Stelle kodiert, in welchem Bereich die Leistungen erbracht wurden. Es gibt hier nur 3 Möglichkeiten:

$A=$ Allgemeinpsychiatrie; $\mathrm{P}=$ Psychosomatik; $\mathrm{K}=$ Kinder- und Jugendpsychiatrie. Falls Sie hier ein F vorfinden sollten, liegt eine Fehler-PEPP vor, beispielsweise falls keine PEPP-relevante ICD-1o Hauptdiagnose kodiert wurde.

Nach der sog. Grouper-Logik von PEPP sollte zunächst geklärt werden, ob ein Fall als voll- oder teilstationär einzuordnen ist. Bei vollstationären Fällen ist zudem 
vorrangig entscheidend, ob die Kriterien für sogenannte Prä-PEPPs erfüllt sind, wobei Prä-PEPPs die am höchsten bewerteten PEPPs darstellen. Um die Prä-PEPPKriterien zu erreichen, wird ein hohes Maß an klinisch indizierter und gut dokumentierter 1:1-Betreuung (bis zu 300 Stunden während eines Aufenthaltes), oder die Erfüllung von mindestens 3 Intensivmerkmalen an $75 \%$ aller Behandlungstage gefordert. Diese Intensivmerkmale werden im OPS-Katalog aufgeführt und später ausführlicher dargestellt. Prä-PEPPs, die mit Po („null“) kodiert werden, stellen die einzigen Strukturkategorien dar, die diagnoseunabhängig vergeben werden.

Tabelle 5 stellt die möglichen Strukturkategorien zusammenfassend dar.

\begin{tabular}{|l|l|}
\begin{tabular}{|l} 
Tab. 5 Strukturkategorien \\
\hline Po
\end{tabular} & Prä-PEPP, nur vollstationär \\
\hline PA & Strukturkategorie Psychiatrie, vollstationär \\
\hline PK & Strukturkategorie Kinder- und Jugendpsychiatrie, vollstationär \\
\hline PP & Strukturkategorie Psychosomatik, vollstationär \\
\hline TA & Strukturkategorie Psychiatrie, teilstationär \\
\hline TK & Strukturkategorie Kinder- und Jugendpsychiatrie, teilstationär \\
\hline TP & Strukturkategorie Psychosomatik, teilstationär \\
\hline PF & Fehler-PEPP und sonstige PEPP \\
\hline
\end{tabular}

Die 3. und 4. Stelle ist in erster Linie diagnoseabhängig. So wird beispielsweise o3 für Schizophrenien oder wahnhafte Störungen oder 15 bei der Hauptdiagnose einer Demenz vom Alzheimer-Typ kodiert. Die ersten vier Stellen kodieren nach dieser PEPP-Notationslogik eine sogenannte Basis-PEPP, beispielsweise PAo3 falls ein Patient mit der Hauptdiagnose Schizophrenie vollstationär in der Erwachsenenpsychiatrie behandelt wurde.

Die 5. Stelle gibt schließlich den Ressourcenverbrauch an, wobei je nach BasisPEPP eine Differenzierung in bis $\mathrm{zu} 4$ Stufen möglich ist, wobei $A=$ höchster; $B=$ zweithöchster $;=$ dritthöchster $;=$ vierthöchster und $Z=$ einen nicht weiter unterteilten Ressourcenverbrauch bedeutet. Steht also an 5 . Stelle ein A bedeutet auch dies eine hohe Vergütung des Aufwandes. Die Kodierung des Ressourcenverbrauchs ist dabei abhängig von sogenannten Kostentrennern.

\section{Was sind wichtige Kostentrenner?}

Nach Analyse der Daten, die von den Kalkulationshäusern an das InEK gesendet wurden, konnte insbesondere die Zuordnung zu verschiedenen Hauptdiagnosen die Kosten der einzelnen Behandlungsfälle mit ausreichender Trennschärfe unterscheiden. Das bedeutet, dass Patienten mit affektiven Störungen sich hinsichtlich der tatsächlich entstehenden Kosten von Patienten mit schizophrenen Störungen oder Abhängigkeitserkrankungen unterscheiden.

Mit dem Begriff Kostentrenner werden demnach die Faktoren bezeichnet, die mit relevanten und nachgewiesenen Kostenunterschieden im Vergleich zu anderen Behand- 
lungsfällen und Verläufen einhergehen. Neben der psychiatrischen Hauptdiagnose sind für Sie wichtige Kostentrenner: das Alter, eine Reihe von komplizierenden somatischen und psychiatrischen Nebendiagnosen sowie Patienten, die eine sehr hohe Betreuungsintensität benötigen oder die Merkmale einer Intensivbehandlung aufweisen. Dabei wird das Alter zumeist in 3 Stufen (64-; 65+; 84+ Jahre) unterteilt.

Während die Zuordnung der Hauptdiagnose nach der Basis-PEPP erfolgt ist, sollten Sie auch in Hinblick auf ihre wirtschaftliche Bedeutung auf eine genaue Dokumentation von komplizierenden somatischen und psychiatrischen Nebendiagnosen achten. Tabelle 6 führt für Sie eine Auswahl von bei psychiatrischen Patienten häufig auftretenden komplizierenden somatischen Nebendiagnosen nach ICD-10 auf.

Tab. 6 Einige wichtige Nebendiagnosegruppen

\begin{tabular}{|c|c|}
\hline A08 & Virusbedingte Darminfektionen \\
\hline A46 & Erysipel \\
\hline B18 & Chronische Virushepatitis \\
\hline B20-B24 & HIV-Krankheit inkl. der Folgen \\
\hline E10-E14 & Diabetes mellitus \\
\hline E51 & Thiaminmangel [Vitamin-B1-Mangel] \\
\hline E66 & Adipositas \\
\hline G20 & Primäres Parkinson-Syndrom \\
\hline G35 & Multiple Sklerose [Encephalomyelitis disseminata] \\
\hline $\mathrm{G} 40-\mathrm{G} 41$ & Epilepsie und Status epilepticus \\
\hline 150 & Herzinsuffizienz \\
\hline$|13-| 18$ & Pneumonien \\
\hline 144 & Sonstige chronische obstruktive Lungenkrankheit \\
\hline 145 & Asthma bronchiale \\
\hline IK25 & Ulcus ventriculi \\
\hline K50 & Crohn-Krankheit [Enteritis regionalis] [Morbus Crohn] \\
\hline $\mathrm{L} 20$ & Atopisches [endogenes] Ekzem \\
\hline M54 & Rückenschmerzen \\
\hline N18 & Chronische Nierenkrankheit \\
\hline R11 & Übelkeit und Erbrechen \\
\hline R42 & Schwindel und Taumel \\
\hline R51 & Kopfschmerz \\
\hline R52 & Schmerz, anderenorts nicht klassifiziert \\
\hline R55 & Synkope und Kollaps \\
\hline
\end{tabular}




\section{Was bedeuten die OPS-Codes?}

Wie bereits kurz dargestellt, stellen neben dem Alter und komplizierenden $\mathrm{Ne}$ bendiagnosen auch Behandlungsfälle mit sehr hoher Betreuungsintensität einen wichtigen Kostentrenner dar. Diese hohe Betreuungsintensität kann mit dem OPS-Code 9.61 (Intensivbehandlung) abgebildet werden, wobei für diese Patienten, wie bereits ausgeführt, entsprechende Prä-PEPPs eingerichtet wurden (Poo3A bis 3D und Poo4Z).

Vereinfacht werden durch die OPS-Codes durchgeführte Therapieeinheiten (TE) von zumeist 25 Minuten von verschiedenen Berufsgruppen abgebildet. Das DIMDI unterscheidet derzeit vier Berufsgruppen, wobei es von Bedeutung und zu kodieren ist, ob die Leistungen von der Gruppe 1 (Ärzte und Psychologen) oder Gruppe 2 (Spezialtherapeuten [Sozialarbeit, Ergotherapie, Physiotherapie, Musiktherapie] und Pflegefachkräfte) erbracht wurden. Zur weiteren Differenzierung der OPS wird entweder der Aufwand in Stunden pro Tag oder die Summe der Behandlungstage, an denen das entsprechende Merkmal vorlag, codiert. Unter die OPS fallen beispielsweise auch die Leistungen in einem besonderen Setting, komplexe Entlassungsplanungen oder eine qualifizierte Entzugsbehandlung.

Die OPS sollen dabei von dem jeweiligen verantwortlichen Leistungserbringer kodiert werden, wobei als Faustformel gilt:

Berufsgruppe + Summe der Therapieeinheiten + Maßnahmen = OPS

Die Tabelle 7 stellt die derzeit wichtigen OPS-Codes in der Erwachsenenpsychiatrie dar.

\section{Tab. 7 OPS-Codes und OPS-Merkmale in der Erwachsenenpsychiatrie}

\begin{tabular}{|c|c|}
\hline $9-60 *$ & Regelbehandlung mit bis zu 2 TE/Woche \\
\hline $9-61^{*}$ & $\begin{array}{l}\text { Intensivmerkmale (Graduierung: } 1-2 ; 3-4 ; 5+\text { ) sind vorhanden (1. besondere Sicherungsmaß- } \\
\text { nahmen; 2. akute Suizidalität oder schweres selbstschädigendes Verhalten; 3. akute Fremdge- } \\
\text { fährdung; 4. schwere Antriebsstörung; 5. keine eigenständige Flüssigkeits-/Nahrungsaufnah- } \\
\text { me; } 6 \text {. akute Selbstgefährdung durch fehlende Orientierung oder Realitätsverkennung; } \\
\text { 7. Entzugsbehandlung mit Vitalgefährdung) }\end{array}$ \\
\hline $9-62^{*}$ & $\begin{array}{l}\text { Psychotherapeutische Komplexbehandlung (es sind mind. } 3 \text { TE/Woche vorhanden, aber keine } \\
\text { Intensivmerkmale) }\end{array}$ \\
\hline $9-640 . *$ & $\begin{array}{l}\text { Erhöhter Betreuungsaufwand entweder als 1:1-Betreuung oder als Kleinstgruppe bis } 3 \text { Perso- } \\
\text { nen (Tagesbezug; akute Fremd- oder Selbstgefährdung) }\end{array}$ \\
\hline $9-641 .^{*}$ & $\begin{array}{l}\text { Krisenintervention (vordringliche, ungeplante, einzeltherapeutische Kontakte außerhalb des } \\
\text { vorgegebenen Therapieplans; tägliche ärztliche Befunderhebung notwendig) }\end{array}$ \\
\hline
\end{tabular}




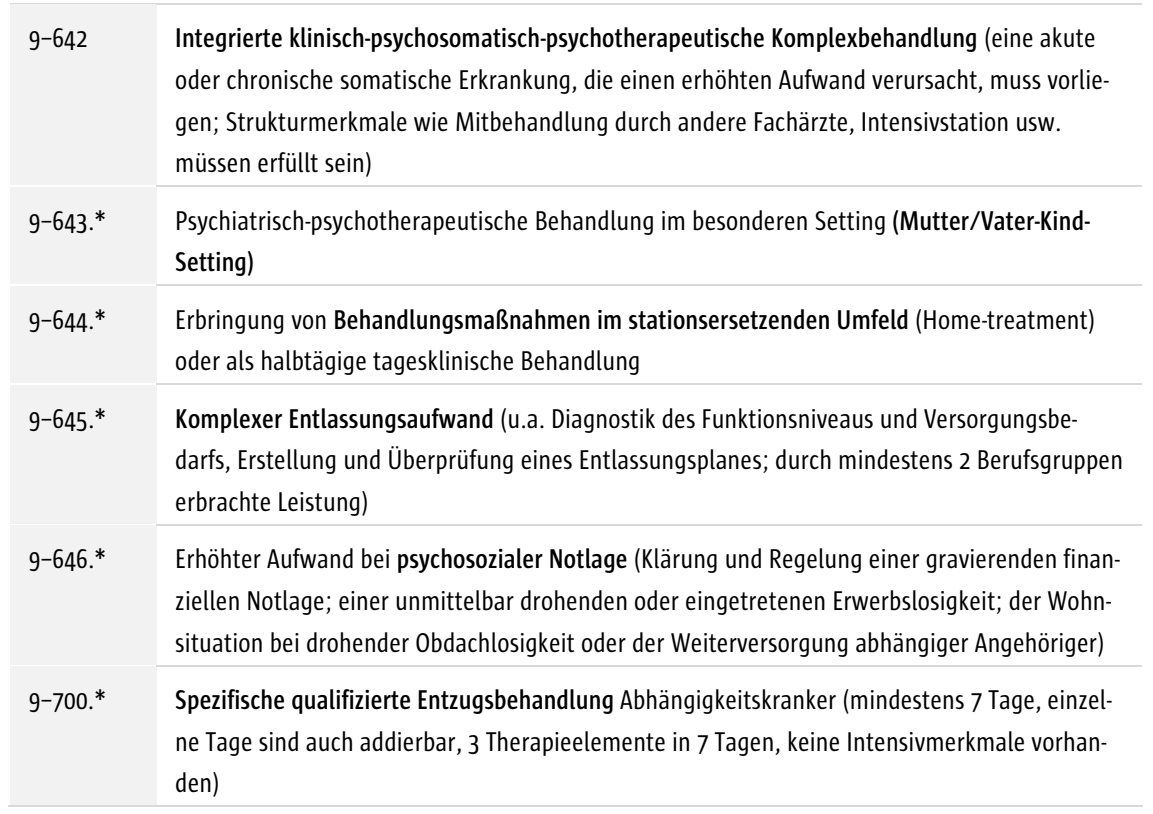

\section{Anwendung der PEPP-Notierung}

Da Sie nun gesehen haben, wie die Notierung von PEPPs mit dem fünfstelligen System aufgebaut ist, wird es Ihnen leichtfallen, die in der Erwachsenenpsychiatrie gebräuchlichen PEPPs zu lesen.

In der Tabelle 8 werden die wichtigsten PEPPs der Erwachsenenpsychiatrie aufgeführt, wobei wir uns der Übersichtlichkeit halber auf die zwei jeweils höchsten Aufwandsstufen (A und B) beschränken:

\section{Tab. 8 Wichtige PEPPs in der Erwachsenenpsychiatrie}

\begin{tabular}{|c|c|}
\hline P003A & $\begin{array}{l}\text { Prä-Strukturkategorie (Prä-PEPP); erhöhter Betreuungsaufwand bei Erwachsenen, 1:1-Betreuung } \\
\text { mit äußerst hohem Aufwand }\end{array}$ \\
\hline P003B & 1:1-Betreuung mit sehr hohem Aufwand \\
\hline P004Z & Intensivbehandlung bei Erwachsenen, ab 3 Intensivmerkmalen, mit sehr hohem Anteil \\
\hline PA01A & Intelligenzstörungen, tief greifende Entwicklungsstörungen, mit komplizierender Konstellation \\
\hline PA01B & wie PA01A; jedoch ohne komplizierende Konstellation \\
\hline PA02A & $\begin{array}{l}\text { Psychische und Verhaltensstörungen durch psychotrope Substanzen, mit Heroinkonsum oder } \\
\text { intravenösem Gebrauch sonstiger Substanzen }\end{array}$ \\
\hline PA02B & $\begin{array}{l}\text { ohne i.v. Gebrauch, jedoch mit komplizierender Konstellation oder mit erhöhter Therapieintensi- } \\
\text { tät oder mit Mutter/Vater-Kind-Setting }\end{array}$ \\
\hline PA03A & $\begin{array}{l}\text { Schizophrenie, oder verwandte Störung, Alter > } 64 \text { lahre oder mit komplizierender Konstellation } \\
\text { oder mit erhöhter Therapieintensität oder mit Mutter/Vater-Kind-Setting }\end{array}$ \\
\hline
\end{tabular}




\begin{tabular}{|c|c|}
\hline PA03B & Alter $<65$ lahre, ohne komplizierende Konstellation \\
\hline PA04A & $\begin{array}{l}\text { Affektive, neurotische, Belastungs-, somatoforme und Schlafstörungen, Alter }>89 \text { lahre oder mit } \\
\text { komplizierender Diagnose und Alter }>64 \text { lahre }\end{array}$ \\
\hline PA04B & $\begin{array}{l}\text { Alter < } 90 \text { Jahre, ohne komplizierende Konstellation, mit Mutter/Vater-Kind-Setting oder mit kom- } \\
\text { plizierender Diagnose oder Alter }>64 \text { Jahre }\end{array}$ \\
\hline PA14A & $\begin{array}{l}\text { Persönlichkeits- und Verhaltensstörungen, Essstörungen und andere Störungen, Alter > } 64 \text { Jahre } \\
\text { oder mit komplizierender Konstellation oder mit erhöhter Therapieintensität }\end{array}$ \\
\hline PA14B & Alter < 65 Jahre, ohne komplizierende Konstellation \\
\hline PA15A & $\begin{array}{l}\text { Organische Störungen, amnestisches Syndrom, Alzheimer-Krankheit und sonstige degenerative } \\
\text { Krankheiten des Nervensystems, mit komplizierender Konstellation oder mit hohem Anteil Inten- } \\
\text { sivbehandlung oder mit erhöhter Therapieintensität }\end{array}$ \\
\hline PA15B & $\begin{array}{l}\text { mit bestimmten Demenzerkrankungen oder mit komplizierender Diagnose oder Alter > } 84 \text { Jahre } \\
\text { oder mit erhöhtem Anteil Intensivbehandlung }\end{array}$ \\
\hline
\end{tabular}

\section{Reaktionen auf die Kritik vor der verpflichtenden Einführung von PEPP}

Ab 2013 konnten Kliniken das neue PEPP optional übend anwenden, wobei sich etwa $15 \%$ aller Kliniken dazu entschieden haben. Ein Hauptkritikpunkt an PEPP war, dass es durch vorzeitige Entlassungen zu einer Verschlechterung der Versorgung insbesondere von schweren psychischen Störungen kommen könnte. Hintergrund dieser Befürchtungen ist, dass vom InEK der Ressourcenverbrauch im Laufe der stationären Behandlungstage eines Patienten degressiv kalkuliert wurde. Es wird also angenommen, dass die Kosten für einen stationären Patienten im Behandlungsverlauf immer mehr abnehmen sollten. Das tagesbezogene Entgelt, dass sich aus der Multiplikation der Bewertungsrelationen der PEPPs (Faktor 3,5 bis Faktor 0,7) mit einem Basisentgeltwert (z.B. 250 Euro) ergibt, würde also bei einer abgestuften Degression der Bewertungsrelationen immer weiter abnehmen.

Nach einer massiven Kritik vor der verpflichtenden Einführung des PEPP ab 2015 sowohl durch Patientenverbände und Fachgesellschaften gleichermaßen (u.a. wurde eine noch mangelnde Differenzierung, nicht ausreichende Abbildung der klinischen Realität und Zeitmangel beim Aufbau der notwendigen IT-Maßnahmen kritisiert) wurde die verpflichtende Erprobungsphase von 2015 auf 2017 verschoben.

Zudem wurde das InEK beauftragt, $\mathrm{zu}$ analysieren, inwiefern alternativ eine tagesbasierte Berechnung ohne Degression die Anreize für zu frühe oder auch zu späte Entlassungen verhindern kann. Diese von der Deutschen Gesellschaft für Psychiatrie und Psychotherapie, Psychosomatik und Nervenheilkunde (DGPPN) vorgeschlagene alternative Kalkulation eines Tagesbezogenen Entgelts für Psychiatrie und Psychosomatik (TEPP) sollte unabhängiger von Diagnosen sich eher an einer tatsächlichen tagesbezogenen Abstufung der psychiatrischen, somatischen und psychosozialen Leistungen orientieren. 


\section{Schlussbemerkung}

Nach aktueller Gesetzgebung wird die Teilnahme am PEPP-System für alle Krankenhäuser ab 2017 verpflichtend werden. Dann beginnt eine fünfjährige Konvergenzphase, in der die Durchschnittsvergütungen (Basisentgelte) eines Krankenhauses schrittweise an ein landesweites Niveau angepasst werden sollen. Voraussichtlich ab 2022 wird ein für alle voll- und teilstationären Leistungen und für die entsprechenden Vergütungsstufen bundeseinheitliches Festpreissystem der Basis-Entgeltwerte gelten. Schlussendlich werden diejenigen Kliniken und psychiatrischen Abteilungen diese Herausforderung meistern, die sich frühzeitig auf ein neues Entgeltsystem vorbereitet haben. Diese Vorbereitung hängt entscheidend von Ihnen als in den Kliniken tätige Ärzte ab, sodass wir hoffen, dass Ihnen dieses Kapitel geholfen hat, mit den "Spielregeln“ des PEPP auf allen Ebenen vertrauter zu sein.

\section{Literaturempfehlung}

MHH Medizinische Hochschule Hannover (2015) Psychiatrie-Entgelt. URL: http://www.psychiatrie-entgelt.de/ (abgerufen am 20.01.2015). Da PEPP als lernendes System angelegt ist, das zumindest jährlich auf Grundlage der Daten aller beteiligten Kliniken angepasst und aktualisiert werden wird, empfehlen wir Ihnen am ehesten, die zukünftigen Veränderungen im Internet zu verfolgen. 